\title{
Judgment Aggregation Rules and Voting Rules
}

\author{
Jérôme Lang ${ }^{1}$ and Marija Slavkovik ${ }^{2}$ \\ ${ }^{1}$ Université Paris-Dauphine, France \\ lang@lamsade.dauphine.fr \\ 2 University of Liverpool, UK, and University of Bergen, Norway \\ marija.slavkovik@infomedia.uib.no
}

\begin{abstract}
Several recent articles have defined and studied judgment aggregation rules based on some minimization principle. Although some of them are defined by analogy with some voting rules, the exact connection between these rules and voting rules is not always obvious. We explore these connections and show how several well-known voting rules such as the top cycle, Copeland, maximin, Slater or ranked pairs, are recovered as specific cases of judgment aggregation rules.
\end{abstract}

\section{Introduction}

Judgment aggregation studies the problem of finding collective judgments that represent a collection of individual judgments on several logically interrelated issues. Originating from law and studied in social choice theory, it has now become clear that judgment aggregation also relates to various fields of knowledge representation, such as belief merging or nonmonotonic reasoning.

The literature on judgment aggregation has, until recently, focused much more on impossibility or possibility theorems than on the study of specific rules, which departs from the (admittedly much older) field of voting theory. However, several recent, independent papers have started to explore the zoo of interesting, concrete judgment aggregation rules, in particular [MO09, EGP12, NPP11, LPSvdT11, DP12, Die12].

Some of these rules were obviously defined by analogy with a well-known voting rule; for instance, the so-called Young rule in [LPSvdT11], that looks for a minimum number of agents to remove so that the resulting profile becomes majority-consistent, is the obvious counterpart of the Young voting rule. For a few others, the analogy remains clear, but the formal connection is less trivial to establish; as an example of such result, [EGP12] show that the distance-based procedure proposed in [MO09] (and close to the distance-based majoritarian merging operator proposed in [KPP02]), corresponds in some sense to the Kemeny rule. For a few other rules, the analogy itself is not obvious.

The formal connection between judgment aggregation rules and voting rules makes use of the preference agenda [DL07]: given a set of alternatives $C$, this agenda is composed of propositions of the form " $x$ is preferred to $y$ ", where $x$ and $y$ are alternatives in $C$; a profile corresponds to a set of individual judgments, whose consistency condition corresponds to the transitivity of the individual votes. A nontrivial question is whether the collective judgment set should be consistent with the transitivity constraint, or only with the constraint expressing the existence of an undominated alternative.

P. Perny, M. Pirlot, and A. Tsoukiàs (Eds.): ADT 2013, LNAI 8176, pp. 230-243 2013.

(C) Springer-Verlag Berlin Heidelberg 2013 
Section 2 introduces the judgment aggregation framework we are using. Section 3 gives some background on judgment aggregation rules, while Section 4 gives some background on voting rules. Section 5 addresses the question of relations between voting and judgment aggregation rules in full detail: we define a formal way of mapping a judgment aggregation rule into two voting rules, obtained by requiring the collective judgment to be consistent with one constraint or the other.

It is rather intriguing to see which pairs of well-known voting rules correspond to the same judgment aggregation rule. For instance, as we show, the Copeland rule comes together with the Slater rule, whereas the maximin rule comes together with the "ranked pairs" rule. Section 6 discusses some implications of our results as well as further research issues.

\section{Judgment Aggregation: General Definitions}

Let $\mathcal{L}$ be a set of well-formed propositional logical formulas, including $\top$ (tautology) and $\perp$ (contradiction). An issue is a pair of formulas $\varphi, \neg \varphi$ where $\varphi \in \mathcal{L}$ and $\varphi$ is neither a tautology nor a contradiction. An agenda $\mathcal{A}$ is built up from a finite set of issues, and has the form $\mathcal{A}=\left\{\varphi_{1}, \neg \varphi_{1}, \ldots, \varphi_{m}, \neg \varphi_{m}\right\}$. The preagenda $[\mathcal{A}]$ associated with $\mathcal{A}$ is $[\mathcal{A}]=\left\{\varphi_{1}, \ldots, \varphi_{m}\right\}$. A judgment on $\varphi \in[\mathcal{A}]$ is one of $\varphi$ or $\neg \varphi$. A judgment set $J$ is a subset of $\mathcal{A}$. It is complete iff for each $\varphi \in[\mathcal{A}]$, either $\varphi \in J$ or $\neg \varphi \in J$.

Constraints can be specified to explicitly represent logical dependencies enforced on agenda issues. Since we have a finite $\mathcal{L}$, without loss of generality we can assume that the constraints consist of one propositional formula (typically the conjunction of several simpler constraints). The constraint associated to an agenda $\mathcal{A}$ is thus a consistent formula $\Gamma \in \mathcal{L}$. When not otherwise specified, $\Gamma$ is the tautology $\top$. Involving constraints in judgment aggregation has already been considered in a few places, such as [DL08, GE13].

A judgment set $J$ (and more generally, a set of propositional formulas) is $\Gamma$-consistent if and only if $J \cup\{\Gamma\} \not \models \perp$. Let $\mathcal{D}(\mathcal{A}, \Gamma)$ be the set of all $\Gamma$-consistent judgment sets (for agenda $\mathcal{A}$ ) and $\mathbb{D}(\mathcal{A}, \Gamma) \subset \mathcal{D}(\mathcal{A}, \Gamma)$ be the set of all judgment sets that are also complete. We omit specifying $\mathcal{A}$ and $\Gamma$ when they are clear from the context.

A profile $P=\left\langle J_{1}, \ldots, J_{n}\right\rangle \in \mathbb{D}^{n}(\mathcal{A}, \Gamma)$ is a collection of complete, $\Gamma$-consistent individual judgment sets. Given $I \subseteq\{1, \ldots, n\}$, the sub-profile $P_{I}$ is the collection $P_{I}=\left\langle J_{i} \mid i \in I\right\rangle$. In the whole paper (except at one place), we assume we have an odd number $n$ of voters.

A sub-agenda is a subset of issues from $\mathcal{A}$, that is, a subset of $\mathcal{A}$ of the form $\left\{\varphi_{j}, \neg \varphi_{j} \mid j \in J\right\}$. A sub-preagenda is a subset of $[\mathcal{A}]$. Given a sub-agenda $Y$, the projection of $J$ on $Y$ is $J^{\downarrow Y}=J \cap Y$. Given a profile $P=\left\langle J_{1}, \ldots, J_{n}\right\rangle$, the projection of $P$ on $Y$ is $P^{\downarrow Y}=\left\langle J_{1}^{\downarrow Y}, \ldots, J_{n}^{\downarrow Y}\right\rangle$. An example is given in Figure 1 For $\varphi \in \mathcal{A}$, the set of agents in $P$ with judgment sets that contain $\varphi$ is $N(P, \varphi)=\#\left\{i \mid \varphi \in J_{i}\right\}$.

An irresolute judgment aggregation rule, for $n$ voters, is a function $F_{\Gamma}: \mathbb{D}^{n} \rightarrow 2^{\mathcal{D}} \backslash\{\emptyset\}$, i.e., $F_{\Gamma}$ maps a profile of complete judgment sets to a nonempty set of judgment sets. When $\Gamma$ is omitted, i.e., when we note $F$ instead of $F_{\Gamma}$, we assume that $F$ is defined for any possible constraint $\Gamma$ ( $F$ then defines a family of judgment 


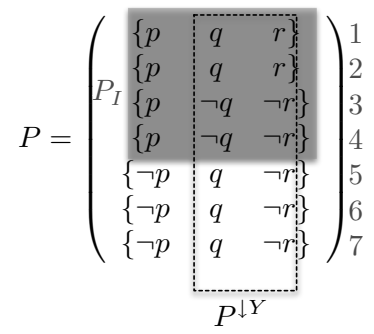

Fig. 1. A profile $P$ for 7 agents for $[\mathcal{A}]=\{p, q, r\}$ and $\Gamma=(p \wedge q) \leftrightarrow r$. The grey shaded area depicts sub-profile $P_{I}$ for $I=\{1,2,3,4\}$, while the dotted lined area corresponds to the projection $P^{\downarrow Y}$ for $Y=\{q, \neg q, r, \neg r\}$. We have, for instance, $N(P, q)=5$.

aggregation rules - one for each $\Gamma$ - but by a slight abuse of language we will still call $F$ a judgment aggregation rule) 1

The majoritarian judgment set associated with profile $P$ contains all elements of the agenda that are supported by a majority of judgment sets in $P$, i.e.,

$$
m(P)=\left\{\varphi \in \mathcal{A} \mid N(P, \varphi)>\frac{n}{2}\right\} .
$$

A profile $P$ is $(\Gamma)$-majority-consistent iff $m(P)$ is $\Gamma$-consistent. A judgment aggregation rule $F_{\Gamma}$ is majority-preserving iff, for every $\Gamma$-majority-consistent profile $P \in \mathbb{D}^{n}, F(P)=\{m(P)\}$.

Given a set of formulas $\Sigma, S \subseteq \Sigma$ is a maximal $\Gamma$-consistent subset of $\Sigma$ if $S$ is $\Gamma$-consistent and no $S^{\prime}$ such that $S \subset S^{\prime} \subseteq \Sigma$ is $\Gamma$-consistent; and $S \subseteq \Sigma$ is a maxcard (for "maximal cardinality") $\Gamma$-consistent subset of $\Sigma$ if $S$ is $\Gamma$-consistent and no $S^{\prime} \subseteq \Sigma$ such that $|S|<\left|S^{\prime}\right|$ is $\Gamma$-consistent. MaxCons $(m(P), \Gamma)$ denotes the set of all maximal $\Gamma$-consistent subsets of $m(P)$. MaxCardCons $(m(P), \Gamma)$ ) denotes the maxcard set of $\Gamma$-consistent subsets of $m(P)$.

\section{Judgment Aggregation Rules}

We recall four minimization-based judgment aggregation rules. We reuse the names from [LPSvdT11] and indicate when a rule has appeared elsewhere with a different name. Let $P=\left\langle J_{1}, \ldots, J_{n}\right\rangle$ from $\mathbb{D}(\mathcal{A}, \Gamma)^{n}$.

Definition 1 (Maximal and maxcard sub-agenda rules). The maximal sub-agenda $(M S A)$ and the maxcard sub-agenda (MCSA) rules are defined as follows:

$$
\begin{array}{r}
M_{S} A_{\Gamma}(P)=\operatorname{Max} \operatorname{Cons}(m(P), \Gamma), \\
M C S A_{\Gamma}(P)=\operatorname{Max} \operatorname{CardCons}(m(P), \Gamma) .
\end{array}
$$

\footnotetext{
${ }^{1}$ We could have opted for the more complex notation $F_{n, \mathcal{A}, \Gamma}$. However, omitting $n$ and $\mathcal{A}$ will not lead to any ambiguity.
} 
The $M S A$ rule is called "Condorcet admissible set", and the $M C S A$ "Slater rule", in [NPP11].

Definition 2 (Ranked agenda). Let $\succsim_{P}$ be the weak order on $\mathcal{A}$ defined by: for all $\psi, \psi^{\prime} \in \mathcal{A}, \psi \succsim_{P} \psi^{\prime}$ iff $N(P, \psi) \geq N\left(P, \psi^{\prime}\right)$. For $\mathcal{A}=\left\{\psi_{1}, \ldots, \psi_{2 m}\right\}$ and a permutation $\sigma$ of $\{1, \ldots, 2 m\}$, let $>_{\sigma}$ be the linear order on $\mathcal{A}$ defined by $\psi_{\sigma(1)}>\ldots>\psi_{\sigma(2 m)}$. We say that $>_{\sigma}$ is compatible with $\succsim_{P}$ if $\psi_{\sigma(1)} \succsim_{P} \ldots \succsim_{P} \psi_{\sigma(2 m)}$. The ranked agenda rule $R A_{\Gamma}$ is defined as $J \in R A_{\Gamma}(P)$ iff there exists a permutation $\sigma$ such that $>_{\sigma}$ is compatible with $\succsim_{P}$ and such that $J=J_{\sigma}$ is obtained by the following procedure:

$$
\begin{array}{ll}
S:=\emptyset ; & 1 \\
\text { for } j=1, \ldots, 2 m \text { do } & 2 \\
\quad \text { if } S \cup\left\{\psi_{\sigma(j)}\right\} \text { is } \Gamma \text {-consistent, then } S:=S \cup\left\{\psi_{\sigma(j)}\right\} & 3 \\
\text { end for; } & 4 \\
J_{\sigma}:=S . & 5
\end{array}
$$

The $R A$ rule is called by the name "leximin rule" by [NPP11].

The next rule is defined as the distance-based rule in [EGP12], "maxweight sub agenda" rule in [LPSvdT11], "Prototype" in [MO09], "median rule" in [NPP11], and "simple scoring rule" in [Die12] has received much more attention that the others. Its relationship to the Kemeny rule is considered in [EGP12] (see also [EM05]).

Definition 3 (Maxweight sub-agenda rule). Let $J \in \mathbb{D}(\mathcal{A}, \Gamma)$. The maxweight subagenda rule $M W A$ is defined as 2

$$
M W A(P)=\arg \max _{J \in \mathbb{D}(\mathcal{A}, \Gamma)} W_{P}(J) \quad \text { where } \quad W_{P}(J)=\sum_{\varphi \in J} N(P, \varphi) .
$$

Definition 4 (Young rule). Let $M S P(P)$ be the set of all maxcard $\Gamma$-majorityconsistent sub-profiles $P_{I} \in \mathbb{D}^{|I|}(\mathcal{A}, \Gamma)$ of $P \in \mathbb{D}^{n}(\mathcal{A}, \Gamma)$, namely,

$$
M S P(P)=\left\{P_{I} \mid \text { there } i s \text { no } I^{\prime} \text { such that }|I|<\left|I^{\prime}\right| \text { and } m\left(P_{I^{\prime}}\right) \in \mathbb{D}(\mathcal{A}, \Gamma)\right\} .
$$

The Young judgment aggregation rule is defined as

$$
Y_{\Gamma}(P)=\left\{m\left(P_{I}\right) \mid P_{I} \in M S P(P)\right\} .
$$

\section{Voting Rules}

Let $C=\left\{x_{1}, \ldots, x_{q}\right\}$ be a set of alternatives. An $n$-voter profile over $C$ (recall that $n$ is assumed to be odd) is a collection $V=\left\langle\succ_{1}, \ldots, \succ_{n}\right\rangle$ of linear orders on $C$, called

${ }^{2}$ Alternatively the rule $M W A$ can be defined as a (Hamming) distance based rule

$$
R^{d_{H}, \Sigma}(P)=\arg \min _{J \in \mathbb{D}(\mathcal{A}, \Gamma)} \sum_{i=1}^{n} d_{H}\left(J_{i}, J\right) .
$$

The equivalence between these two definitions was shown in [LPSvdT11]. 
votes. An irresolute voting rule (or voting correspondence) is a function $R$ mapping every profile $V$ into a nonemptyset of alternatives $R(V) \in 2^{C} \backslash\{\emptyset\}$. For every pair of alternatives $(x, y) \in C$ and profile $V$, let $n_{V}(x, y)$ be the number of votes in $V$ ranking $x$ above $y$, and let $M(V)$ be the majority graph associated with $V$, whose vertices are $C$ and containing edge $(x, y)$ iff $n_{V}(x, y)>\frac{n}{2}$. The alternative $x \in C$ is a Condorcet winner for $V$ if there is an outgoing edge in $M(V)$ from $x$ to every $y \neq x$.

We now define several (irresolute) voting rules.

The Top-cycle (TC) rule maps every profile $V$ to the set of alternatives $x \subseteq C$ such that for all $y \in C \backslash x$, there exists a path in $M(V)$ that goes from $x$ to $y$. Equivalently, $T C(P)$ is the smallest set $S$ such that for every $x \in S$ and $y \in C \backslash S$, we have $(x, y) \in M(V)$.

A Slater order for $V$ is a linear order $\succ$ over $C$ maximizing the number of $(x, y)$ s.t. $x \succ y$ iff $(x, y) \in M(V)$. The Slater rule maps a profile $V$ to the set of all alternatives that are dominating in some Slater order for $M(V)$.

The Copeland rule maps $V$ to the set of alternatives maximizing the number $n_{c}(x)$ of outgoing edges from $x$ in $M(V)$.

The ranked pairs rule [Tid87] is defined as follows. We define first its non-neutral version: given a tie-breaking priority, that is, a linear order $\rho$ over $\left\{(x, y) \in C^{2}, x \neq y\right\}$, the linear order $>_{\rho}$ on $\left\{(x, y) \in C^{2}, x \neq y\right\}$ is constructed as follows: $(x, y)>_{\rho}$ $\left(x^{\prime}, y^{\prime}\right)$ iff either (a) $n_{V}(x, y)>n_{V}\left(x^{\prime}, y^{\prime}\right)$ or (b) if $n_{V}(x, y)=n_{V}\left(x^{\prime}, y^{\prime}\right)$ and $\rho$ gives priority to $(x, y)$ over $\left(x^{\prime}, y^{\prime}\right)$. Then all pairs $(x, y)$ are considered in sequence according to $>_{\rho}$, and we build a linear order $\succ_{\rho}$ over $C$ starting with the pair on top of $>_{\rho}$, and iteratively adding the current pair to $\succ_{\rho}$ if it does not make it cyclic. The ranked pairs winner for $V$ according to $\rho$ is the unique undominated element in $\succ_{\rho}$. Now, $x$ is a winner of the neutral ranked pairs rule for $V$ iff it is a winner of the non-neutral ranked pairs rule for some $\rho$. (See [BF12] for a recent discussion on neutral and non-neutral variants of ranked pairs.)

The maximin rule maps $V$ to the set of alternatives that maximize

$$
m m(x, V)=\min _{y \in C \backslash\{x\}} n_{V}(x, y) .
$$

Let $S_{Y}(x, V)$ be the minimal number of votes whose removal from $V$ makes $x$ a Condorcet winner. The Young (voting) rule maps $V$ to the set of alternatives that minimize $S_{Y}(x, V)$.

\section{From Judgment Aggregation to Voting Rules}

In this Section, we assume that judgment profiles contain an odd number $n$ of individual judgments. The reason for this assumption is that the connections to voting rules are much easier to state, and more natural, under this assumption.

A specific type of agenda is the preference agenda associated with a set of alternatives $C$ [DL07] whose propositions are of the form $x P y$ (" $x$ preferred to $y$ ").

Definition 5. The preference agenda associated with $C=\left\{x_{1}, \ldots, x_{q}\right\}$ is $\mathcal{A}_{C}=\left\{x_{i} P x_{j} \mid 1 \leq i<j \leq q\right\}$. 
When $j>i, x_{i} P x_{j}$ is not a proposition of $\mathcal{A}_{C}$, but we will write $x_{j} P x_{i}$ as a shorthand for $\neg\left(x_{j} P x_{i}\right)$.

Definition 6. Let $V=\left\langle\succ_{1}, \ldots, \succ_{n}\right\rangle$ be an $n$-voter profile over $C$. With every individual vote $\succ_{i}$ we associate the individual judgment set

$$
J\left(\succ_{i}\right)=J_{i}=\left\{x P y \mid x \succ_{i} y, \text { for } x, y \in C\right\} .
$$

The judgment aggregation profile associated with $V$ is $P(V)=\left\langle J_{1}, \ldots, J_{n}\right\rangle$.

Conversely, given a judgment set $J$ on $\mathcal{A}_{C}$, the binary relation $\succ_{J}$ over $C$ is defined by: for all $x_{i}, x_{j} \in C, x_{i} \succ_{J} x_{j}$ if $x_{i} P x_{j} \in J$ and $x_{j} \succ_{J} x_{i}$ if $\neg x_{i} P x_{j} \in J$.

Now we define two preference constraints: the transitivity constraint $\operatorname{Tr}$ and the dominating alternative, or "winner", constraint $W$.

Definition 7. We define the transitivity $T r$ and dominating alternative $W$ constraints:

- $\operatorname{Tr}=\bigwedge_{i, j, k \in\{1, \ldots, m\}}\left(\left(x_{i} P x_{j}\right) \wedge\left(x_{j} P x_{k}\right) \rightarrow\left(x_{i} P x_{k}\right)\right)$

- $W=\bigvee_{i \leq m} \bigwedge_{j \neq i}\left(x_{i} P x_{j}\right)$

For complete judgment sets, $\operatorname{Tr}$ is stronger than $W$, therefore, any complete $T r$ consistent judgment set is also $W$-consistent.

Lemma 1. Let $J$ be a judgment set on $\mathcal{A}_{C}$.

- J is Tr-consistent iff $\succ_{J}$ is acyclic;

- J is $W$-consistent iff $\succ_{J}$ has at least one undominated element.

The proof is almost straightforward from Definition 6. $J$ is $\operatorname{Tr}$-consistent if $\succ_{J}$ can be completed into a transitive order, i.e., iff $\succ_{J}$ is acyclic; $J$ is $W$-consistent if some $x$ can be made a winner by adding the missing propositions $x P y$, which is possible iff some $x$ is undominated in $\succ_{J}$.

As a consequence of Lemma 1, any $\operatorname{Tr}$-consistent judgment is also $W$-consistent. Note also that $\succ_{J}$ is a linear order if and only if $J$ is complete and $\operatorname{Tr}$-consistent.

For instance, let $J=\{a P b, a P c, b P c, d P b, c P e, e P b\}$; then

$$
\succ_{J}=\{(a, b),(a, c),(b, c),(d, b),(c, e),(e, b)\}
$$

$J$ is not $T r$-consistent because $b P c \wedge c P e \wedge T r \models \neg e P b$ (or equivalently, $\succ_{J}$ contains the cycle $b \succ_{J} c \succ_{J} e \succ_{J} b$ ). However, it is $W$-consistent: $a$ and $d$ are both undominated in $\succ J$.

For each $x \in C$ we define $W(x)=\bigwedge_{y \in C, y \neq x}(x P y)$. Note that $W$ is equivalent to $\bigvee_{x \in C} W(x)$ and that $J$ is $W(x)$-consistent iff $x$ is undominated in $\succ_{J}$.

Since each vote $\succ_{i}$ is a linear order, the individual judgment sets $J_{i}$ are complete and consistent with $\operatorname{Tr}$ (and a fortiori with $W$ ). The collective judgment will sometimes be required to be consistent with respect to $T r$ and sometimes only to be consistent with respect to $W$. Lemma2 is straightforward from Definition 6 .

Lemma 2. Given a voting profile $V$, for all $x, y \in C, x P y$ is in $m(P(V))$ iff $(x, y) \in M(V)$. 
Proposition 1. A voting profile $V$ has a Condorcet winner iff $m(P(V))$ is $W$ consistent.

Proof. From Lemma $2 x P y$ is in $m(P(V))$ iff $M(V)$ contains $(x, y)$. Since $n$ is odd, $m(P(V))$ contains either $x_{i} P x_{j}$ or $x_{j} P x_{i}$ for all $i \neq j$, therefore $m(P(V)) \cup\{W\} \not \models \perp$ iff there exists $x \in C$ s.t. $m(P(V))$ contains $\{x P y \mid y \neq x\}$, i.e., , by Lemma 2 again, iff $V$ has a Condorcet winner.

Note that for an even $n, W$-consistency would be equivalent to the existence of a weak Condorcet winner.

Definition 8. Let

$$
W \operatorname{in}(J)=\{x \mid J \cup W(x) \not \models \perp\}
$$

Let $\Gamma \in\{T r, W\}$ and $F$ be a judgment aggregation rule. The voting rule $R_{F, \Gamma}$ induced from $F$ and $\Gamma$ is defined as $x \in R_{F, \Gamma}(P(V))$ if there is a $J \in R_{F, \Gamma}(P(V))$ such that $x \in W$ in $(J)$, or equivalently:

$$
R_{F, \Gamma}(P)=\bigcup_{J \in F_{\Gamma}(P(V))} \operatorname{Win}(J) .
$$

Note that $J \cup\{W\} \not \models \perp$ or $J \cup\{\operatorname{Tr}\} \not \models \perp$, then $W i n(J) \neq \emptyset$, therefore Definition 8 is well-founded.

Thus, for every judgment aggregation rule $F$ we have two voting rules, obtained by requiring the collective judgment set to be acyclic, i.e., consistent with $\mathrm{Tr}$, or to have a undominated element, i.e., consistent with $W$.

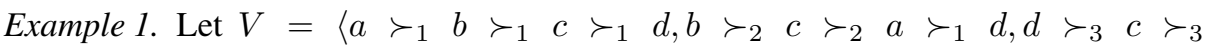
$\left.a \succ_{3} b\right\rangle$. We have $P(V)=\left\langle J_{1}, J_{2}, J_{3}\right\rangle$ with $J_{1}=\{a P b, a P c, a P d, b P c, b P d, c P d\}$, $J_{2}=\{b P a, b P c, b P d, c P a, c P d, a P d\}$ and $J_{3}=\{d P a, d P b, d P c, c P a, c P b, a P b\}$; and we have $m(P(V))=\{a P b, b P c, c P a, a P d, b P d, c P d\}$.

Let us choose $F=M S A$ and $\Gamma=T r$.

We have $F_{T r}(P(V))=\left\{J, J^{\prime}, J^{\prime \prime}\right\}$, where $J=\{a P b, b P c, a P d, b P d, c P d\}$, $J^{\prime}=\{a P b, c P a, a P d, b P d, c P d\}$ and $J^{\prime \prime}=\{b P c, c P a, a P d, b P d, c P d\}$.

Now, $\operatorname{Win}(J)=\{a\}, \operatorname{Win}\left(J^{\prime}\right)=\{c\}$ and $\operatorname{Win}\left(J^{\prime \prime}\right)=\{b\}$.

Therefore, $R_{M S A, T r}(P(V))=\operatorname{Win}(J) \cup \operatorname{Win}\left(J^{\prime}\right) \cup \operatorname{Win}\left(J^{\prime \prime}\right)=\{a, b, c\}$.

\section{Proposition 2.}

1. $R_{M S A, T r}=$ TopCycle

2. $R_{M S A, W}=\left\{\begin{array}{l}\{c\} \text { if } V \text { has a Condorcet winner } c \\ C \quad \text { otherwise }\end{array}\right.$

Proof. We prove the first correspondence. From Lemmas 1 and $2, J \in \operatorname{Max} C o n s(m$ $(P), T r)$ iff $\succ_{J}$ is a maximal acyclic sub-graph of $M(V)$. Let $x \in T C(V)$; then there exists an acyclic subrelation $G$ of $M(V)$ containing, for all $y \neq x$, a path from $x$ to $y$. $G$ can be completed into a maximal acyclic subrelation $G^{\prime}$ of $M(V)$, and $x$ is undominated in $G^{\prime}$ (because adding an edge to any $y \neq x$ would create a cycle), therefore $G^{\prime}$ corresponds to a maximal $T r$-consistent subset $J$ of $m(P(V))$, consistent with $W(x)$, 
which means that $x \in R_{M S A, T r}(V)$. Conversely, if there is a $J \in R_{M S A, T r}(V)$ such that $x \in W i n(J)$, then $\succ_{J}$ is a maximal acyclic subrelation of $M(V)$ in which $x$ does not have any incoming edge. Assume $x \notin T C(V)$; then there is an $y$ such that there is no path from $x$ to $y$ in $M(V)$. Obviously, $(x, y) \notin M(V)$, therefore, since $M(V)$ is complete, $(y, x) \in M(V)$. Adding $(y, x)$ to $\succ_{J}$ results in an acyclic subrelation of $M(V)$ that contains $\succ_{J}$, therefore $\succ_{J}$ is not a maximal acyclic subset of $M(V)$, contradiction.

Now we prove the second correspondence. Assume there is no Condorcet winner. Let $x \in C$. Let $S(x)$ be the subset of $m(P(V))$ defined by $\{y P z \mid z \neq x, y P z \in m(P(V))\} . S(x)$ is $W$-consistent, because it is consistent with $W(x)$. Assume $S(x)$ is not maximal: then there is some element of $m(P(V)) \backslash S(x)$ that can be added to $S(x)$ without violating $W$-consistency; now, every element of $m(P(V)) \backslash S(x)$ is of the form $y P x$. Let $S^{\prime}=S(x) \cup\{y P x\} . S^{\prime}$ is not consistent with $W(x)$. Therefore, since it is $W$-consistent, it must be consistent with $W(z)$ for some $z \neq x$. This implies that there is no $t P z \in S^{\prime}$, therefore, no $t P z \in S(x)$. Now, by construction of $S(x)$, this means that there is no $t P z \in m(P(V))$, which implies that $z$ is a Condorcet winner: contradiction.

\section{Proposition 3.}

1. $R_{M C S A, T r}=$ Slater

2. $R_{M C S A, W}=$ Copeland

Proof. For point 1 , let $J \in M C S A_{T r}(P(V))$, hence $J \in \operatorname{Max} \operatorname{CardCons}(m(P), T r)$ and $\succ_{J}$ is an acyclic subrelation of $M(V)$. Let $>$ be a linear order extending $\succ_{J}$. The number of edge reversals needed to obtain $>$ from $\succ_{J}$ is $|m(P(V)) \backslash J|$. This number is minimal iff $J$ has a maximal cardinality. Consequently, $>$ is a Slater order for $V$. Conversely, let $>$ be a Slater order for $V$ and let $J=\{x P y \mid x>y$ and $x P y \in m(P(V))\}$. Because $>$ is a linear order, $J$ is $T r$ consistent. Moreover, $|m(P(V)) \backslash J|$ is the number of edge reversals needed to obtain $>$ from $M(V)$. Since $|m(P(V)) \backslash J|$ is minimal, $|J|$ is maximal and therefore $J \in M C S A_{T r}(P(V))$. This one-to-one correspondence between Slater orders for $V$ and maxcard acyclic subgraphs of $P(V)$ allows us to conclude.

For point 2, let $J \in \operatorname{Max} \operatorname{CardCons}(m(P), W)$. From $J \cup\{W\} \not \models \perp$ it follows that there exists a $x \in C$ s.t. for every $y \in C, y P x \notin J$. For every $y \in C$, consider $z \in C, z \neq x$, such that $y P z \in m(P(V))$. Adding $y P z$ to $J$ results in a judgment set which is still $W$-consistent, therefore the maximum $W$-consistent subsets of $m(P(V))$ are of the form $J_{x}=m(P(V)) \backslash\{y P x, y \neq x\}$ for some $x \in C$, and such a judgment set $J_{x}$ is a maxcard $W$-consistent subset of $m(P(V))$ iff $|\{y \mid x P y \in m(P(V))\}|$ is maximal, i.e., using Lemma 2, iff $x \in \operatorname{Copeland}(V)$.

Example 2. Let $V$ be such that $M(V)=\{(\mathrm{a}, \mathrm{b}),(\mathrm{a}, \mathrm{c}),(\mathrm{b}, \mathrm{c}),(\mathrm{b}, \mathrm{d}),(\mathrm{c}, \mathrm{d}),(\mathrm{d}, \mathrm{a})\}$, i.e., $m(P(V))=\{a P b, a P c, b P c, b P d, c P d, d P a\}$. The only maxcard $T r$-consistent subset of $m(P(V))$ is $J=\{a P b, a P c, b P c, b P d, c P d\}$, and $W i n(J)=\{a\} ; a$ is also the only Slater winner for $P$. Now, $m(P(V))$ has two maxcard $W$-consistent subsets: $J$ and $J^{\prime}=\{a P c, b P c, b P d, c P d, d P a\} ; \operatorname{Win}\left(J^{\prime}\right)=\{b\} ; a$ and $b$ are also the Copeland winners for $V$. 


\section{Proposition 4.}

1. $R_{R A, T r}=$ ranked pairs.

2. $R_{R A, W}=$ maximin.

Proof. The proof of point (1) is simple, due to the similarity of the definitions of ranked pairs and $R A$, and observing that adding $x P y$ to a current $T r$-consistent judgment set without violating $\operatorname{Tr}$ corresponds to adding $(x, y)$ to a current acyclic graph without creating a cycle. The proof of point (2) is more interesting. The candidate $x$ is a maximin winner if it maximizes $m m(x, V)$, or equivalently, if it $\operatorname{minimizes} \max _{y} n_{V}(y, x)$. Let $\beta=\min _{x} \max _{y} n_{V}(y, x)$. (Note that we have $\beta \geq \frac{1}{2}$ when there is no Condorcet winner.) Assume that $x$ is a Maximin winner for $V$. In order to show that $x \in R_{R A, W}(V)$, we have to construct a linear order $\succ=\succ_{\sigma}$ on $\left\{x P y \mid(x, y) \in C^{2}, x \neq y\right\}$, compatible with $\succsim_{P}$, such that the judgment set $J_{\sigma}$ obtained by following $\succ_{\sigma}$ is such that $x \in \operatorname{Win}\left(J_{\sigma}\right)$. Let $\succ_{\sigma}$ be as follows:

1. the first propositions of $\succ_{\sigma}$ are all $u P v$ such that $n_{V}(u, v)>\beta$, with ties broken in an arbitrary manner;

2. the propositions that follow in $\succ_{\sigma}$ are all $y P z$ such that $n_{V}(y, z)=\beta$ and $z \neq x$;

3. the following propositions are all $y P x$ such that $n_{V}(y, x)=\beta$;

4. the rest of $\succ_{\sigma}$ does not matter.

We now follow step by step the construction of $J_{\sigma}$. During step (1) - corresponding to considering one by one the proposition in (1) above - we consider all the propositions $u P v$ such that $n_{V}(u, v)>\beta$, and all are added to $S$, because the resulting judgment set is consistent with $W(x)$, and a fortiori with $W$ (otherwise it would be the case that for all $y, n_{V}(y, x)>\beta$, contradicting $\min _{x} \max _{y} n_{V}(y, x)=\beta$ ). During step (2) all propositions $y P z$ such that $n_{V}(y, z)=\beta$ and $z \neq x$ are considered one by one, and they are all added to $S$, because the resulting judgment set is, each time, consistent with $W(x)$ and a fortiori with $W$. After steps (1) and (2), due to the fact that $\beta=\min _{x} \max _{y} n_{V}(y, x), S$ contains some $y P z$ for all $z \neq x$. Step (3) considers all $y P x$ such that $n_{V}(y, x)=\beta$, and does not add them to $S$, because this would make it inconsistent with $W$. Finally, the propositions considered in Step (4) are not of the form $y P x$. Therefore, $x \in W i n\left(J_{\sigma}\right)$ and $x \in R_{R A, W}(V)$.

Conversely, let $x \in R_{R A, W}(V)$. Let $>$ be the order refining $\succsim_{P}$ such that the judgment set obtained is $J$, with $x \in \operatorname{Win}(J)$. First, all formulas $u P v$ such that $N(P, u P v)>\beta$ are added to $S$ without creating any inconsistency with $W$. Then, $>$ must consider all propositions $z P y$ such that $N(P, z P y)=\beta$ and $y \neq x$, and add them all to $S$; at this point, for any $y \neq x$, a proposition $z P y$ has been considered and added to $S$, otherwise there would be an $y$ such that for no $z$ it holds that $n_{V}(z, y) \geq \beta$, which would contradict $\beta=\min _{x} \max _{y} n_{V}(y, x)$. Therefore, no propositions $z P x$ will be added to $S$ (or else $W$ would be violated). Therefore, $x$ is such that $\min _{x} \max _{y} n_{V}(y, x) \leq \beta$, hence $\min _{x} \max _{y} n_{V}(y, x)=\beta: x$ is a maximin winner. 
Example 3. Let $n=9$ and $V$ such that $n_{V}$ is as follows:

$$
\begin{array}{c|c|c|c|c}
n_{V} & a & b & c & d \\
\hline a & - & 6 & 2 & 4 \\
\hline b & 3 & - & 5 & 6 \\
\hline c & 7 & 4 & - & 2 \\
\hline d & 5 & 3 & 7 & -
\end{array}
$$

The weak order $\succsim_{P}$ starts with $c P a$ and $d P c$ (tied), then $a P b$ and $b P d$, then $b P c$ and $d P a$, etc. Applying $R A$ with $\Gamma=W$ starts by adding $c P a$ and $d P c$, whatever the choice of the linear order $\succ_{\sigma}$ refining $\succsim_{P}$. Next, there is a choice between $a P b$ or $b P d$. If $a P b$ is considered first (that is, if $a P b \succ_{\sigma} b P d$ ), then it is added to $S, b P d$ is not (because it would violate $W$-consistency), and then all other propositions except $a P d, b P d$ and $c P d$ are added. The other choice is similar, replacing $d$ by $b$. Therefore, $R A(P(V), W)$ contains the two judgment sets

$$
J_{1}=\{d P a, d P B, d P c, a P b, b P a, a P c, c P a, b P c, c P b\}
$$

and

$$
J_{2}=\{b P a, b P c, b P d, a P c, c P a, a P d, d P a, c P d, d P a\},
$$

with $\operatorname{Win}\left(J_{1}\right)=d$ and $\operatorname{Win}\left(J_{2}\right)=b$. We check that $b$ and $d$ are also the maximin winners for $V$.

Applying $R A$ with $\Gamma=W$ first adds $c P a$ and $d P c$. Next, there is a choice between $a P b$ or $b P d$. If $a P b$ is considered first, then it is added to $S, b P d$ is not, then all other propositions except $a P d, b P d$ and $c P d$ are added.

For $M W A$, it is already known that the choice of the transitivity constraint leads to the Kemeny rule, i.e., $R_{M W A, T r}=$ Kemeny. The proof can be found in [EGP12].

The choice of the $W$ constraint leads to an unknown voting rule, for which, interestingly, the winners maximizes the sum of the Borda score and a second term: if $S_{B}(P, x)$ is the Borda score of $P$ for profile $P, R_{M W A, W}$ is the voting rule defined by

$$
S_{M W A, W}(x)=S_{B}(P, x)+\sum_{y \neq z \neq x} \max \left(N_{P}(y, z), N_{P}(z, y)\right)
$$

and

$$
R_{M W A, W}(P)=\underset{x \in X}{\operatorname{argmax}} S_{M W A, W}(x) .
$$

We give in Appendix an example of winner determination for this rule, which shows that it differs from Borda.

The connection between the Young judgment aggregation rule and the Young voting rule is less clear that is seems at first glance: because the removal of judgments (and votes) can make the number of judgments (votes) even, the voting rule obtained from $Y$ together with $W$ is not Young (even for $n$ odd), but a weak version of Young: the weak Young voting rule is defined as the voting rule, except that we look for a minimal number of votes whose removal in $V$ makes $x$ a weak Condorcet winner (where $x$ is a weak Condorcet winner if for any $y \neq x$, at least half of the voters prefer $x$ to $y$ ). 
Proposition 5. $R_{Y \text { oung,W }}=$ WeakYoung.

Proof. Removing a minimal number of judgments from $P(V)$ so as to make it consistent is equivalent to removing a minimal number of votes from $V$ so that the majority graph contains an undominated outcome, i.e., so that there exists a weak Condorcet winner.

$R_{Y \text { oung,Tr }}$ does not appear to be a known voting rule. It consists of the dominating candidates in maximum cardinality sub-profiles of $P(V)$ whose majoritarian aggregation is acyclic.

Another judgment aggregation rule defined in [LPSvdT11] is the distance-based rule $R_{d_{H}, \max }$. Because the voting rules we obtain from it are not known voting rules, we omit the corresponding results.

\section{Discussion}

We have obtained a number of correspondences between judgment aggregation rules and pairs of voting rules. It is especially interesting to see which pairs come together. We summarize the results here.

\begin{tabular}{c||c|c|c|c|c|}
$R_{F, \Gamma}$ & $F=M S A$ & $F=M C S A$ & $F=M W A$ & $F=R A$ & $F=Y$ \\
\hline$\Gamma=T r$ & Top Cycle & Slater & Kemeny & ranked pairs & weak Young \\
\hline$\Gamma=W$ & & Copeland & & maximin &
\end{tabular}

Note that if the assumption that profiles have an odd number of judgments sets is relaxed, then the voting rules obtained are generally be weak versions of the usual voting rule, strict majority being replaced by weak majority. In particular, $R_{M C S A, W}$ would be Copeland ${ }^{0}$, where ties count as much as victories.

What do these results tell us? After all, these judgment aggregation rules have not been widely studied yet (although some of them have been introduced independently in several papers), and one may argue that they were defined in such a way that their specialization to the preference agenda correspond to such or such voting rule, and one may advocate that this makes these correspondence results rather pointless. This is an important point: while the premise is not entirely false (at least for some of the rules, such as RA), we would strongly disagree with the conclusion. The definition and study of judgment aggregation rules is only starting, and knowing that a judgment aggregation rule specializes to a well-known voting rules (sometimes, to two well-known voting rules) is a hint that the judgment aggregation rule is a natural generalization of interesting voting rules, which is a first justification for studying it. Also, it gives insights about the properties it may satisfy. In particular, a challenging question is the axiomatization of judgment aggregation rules, and for this, a good start could be to start with the axiomatization (when it exists) of the voting rule(s) into which the judgment aggregation rule degenerates.

In our correspondence results, a voting rule is defined from two elements: a judgment aggregation operator and a constraint. This is reminiscent of a recent research stream on the distance rationalizability of voting rules (see [Bai87, Kla05a, Kla05b, MN08] 
for early works and [EFS09, EFS12] for a systematic study). There, one seeks to define voting rules via a distance between profiles and a consensus class. In some sense, our judgment aggregation rules play the role of distances whereas the constraint plays the role of the consensus class. More precisely, the $\operatorname{Tr}$ and $W$ constraint more or less correspond to, respectively, the strong unanimity and Condorcet consensus classes, with a noticeable difference: the definition of a consensus class bears on a profile, whereas a constraint bears on an (individual or collective) judgment set (this may explains why we don't have any constraint that corresponds to the unanimity and majority consensus classes). This is consistent with the fact that the two rules we obtain by letting $\Gamma=W$ are also rationalizable for the Condorcet consensus class (see [Kla05b] for Copeland and [EFS09] for maximin).

The discussion about distance rationalizability leads to a very intriguing question. One of the key questions in [EFS12] is a systematic study of which rules can be axiomatized by a given consensus class or a given distance. They not only show that some rules are indeed axiomatizable via a given consensus class or a given distance function, but also that some rules are not. This leads us to ask the following question: which voting rules are definable from a judgment aggregation rule by specializing to the preference agenda and imposing the $\operatorname{Tr}$ or the $W$ constraint? Asked this way, this question is trivial; the judgment aggregation rule can be defined such as its application to the preference agenda behaves exactly like the voting rule we started from. However, suppose we ask the judgment aggregation rule to be neutral with respect to propositional symbols, which means that if $\sigma$ is a permutation of the set of propositional symbols, $J_{\sigma}$ the judgment set obtained by applying $\sigma$ in every $\varphi \in J$ and $P(V)_{\sigma}=\left\{J_{\sigma} \mid J \in P(V)\right\}$, then $F\left(P(V)_{\sigma}\right)=F(P(V))_{\sigma}$. (Note that this is the case of all the rules we study here.) Then the question becomes highly nontrivial, and we suspect that some well-known rules will not be definable this way.

Finally, as argued three paragraphs above, the definition of judgment aggregation rules which specialize to well-known voting rules (and thereby give them a justification) is a bottom-up process. A subsequent top-down process would consists in applying these judgment aggregation rules (obtained as a generalization from voting rules) to other specific agendas and/or with other constraints. We give here two examples. A first example would consist in keeping the preference agenda and to consider constraints that are intermediate between $\operatorname{Tr}$ and $W$, such as the judgment set being transitive on the top $k$ candidates; for instance, $k=2$ this would be $\bigvee_{x \neq y}\left(\bigwedge_{z \neq x} x P z \wedge \bigwedge_{z \neq x, y} y P z\right)$. A second example would consist in keeping an preference agenda of the form $\{x P y, x, y \in X\}$, but with a very different meaning, where $x P y$ means that $x$ and $y$ are in the same equivalence class, and choose $\Gamma$ as the expression of an equivalence relation; this process will give interesting rules for aggregating equivalence relations.

Acknowledgements. We thank Gabriella Pigozzi, Leon van der Torre and Srdjan Vesic for helpful discussions and comments, as well as the anonymous ADT-13 referees for their useful reviews. 


\section{References}

[Bai87] Baigent, N.: Metric rationalization of social choice functions according to principles of social choice. Mathematical Social Science 14(1), 59-65 (1987)

[BF12] Brill, M., Fischer, F.A.: The price of neutrality for the ranked pairs method. In: AAAI (2012)

[Die12] Dietrich, F.: Scoring rules for judgment aggregation. MPRA paper, University Library of Munich, Germany (2012)

[DL07] Dietrich, F., List, C.: Arrow's theorem in judgment aggregation. Social Choice and Welfare 29(1), 19-33 (2007)

[DL08] Dietrich, F., List, C.: Judgment aggregation under constraints. In: Boylan, T., Gekker, R. (eds.) Economics, Rational Choice and Normative Philosophy, Routledge (2008)

[DP12] Duddy, C., Piggins, A.: A measure of distance between judgment sets. Social Choice and Welfare 39, 855-867 (2012)

[EFS09] Elkind, E., Faliszewski, P., Slinko, A.: On distance rationalizability of some voting rules. In: TARK, pp. 108-117 (2009)

[EFS12] Elkind, E., Faliszewski, P., Slinko, A.: Rationalizations of condorcet-consistent rules via distances of hamming type. Social Choice and Welfare 39(4), 891-905 (2012)

[EGP12] Endriss, U., Grandi, U., Porello, D.: Complexity of judgment aggregation. Journal Artificial Intelligence Research (JAIR) 45, 481-514 (2012)

[EM05] Eckert, D., Mitlöhner, J.: Logical representation and merging of preference information. In: Proceedings of the IJCAI 2005 Multidisciplinary Workshop on Preference Handling (2005)

[GE13] Grandi, U., Endriss, U.: Lifting integrity constraints in binary aggregation. Artificial Intelligence, 199-200, 45-66 (2013)

[Kla05a] Klamler, C.: Borda and condorcet: some distance results. Theory and Decision 59(2), 97-109 (2005)

[Kla05b] Klamler, C.: The copeland rule and condorcet's pirnciple. Economic Theory 25(3), 745-749 (2005)

[KPP02] Konieczny, S., Pino-Pérez, R.: Merging information under constraints: a logical framework. Journal of Logic and Computation 12(5), 773-808 (2002)

[LPSvdT11] Lang, J., Pigozzi, G., Slavkovik, M., van der Torre, L.: Judgment aggregation rules based on minimization. In: TARK, pp. 238-246 (2011)

[MN08] Meskanen, T., Nurmi, H.: Closeness counts in social choice. In: Power, Freedom, and Voting, pp. 289-306. Springer (2008)

[MO09] Miller, M.K., Osherson, D.: Methods for distance-based judgment aggregation. Social Choice and Welfare 32(4), 575-601 (2009)

[NPP11] Nehring, K., Pivato, M., Puppe, C.: Condorcet admissibility: Indeterminacy and path-dependence under majority voting on interconnected decisions (July 2011), http://mpra.ub.uni-muenchen.de/32434/

[Tid87] Tideman, T.N.: Independence of clones as a criterion for voting rules. Social Choice and Welfare 4, 185-206 (1987) 


\section{Appendix}

\section{$R_{M W A, W}$}

Let $X=\{a, b, c, d, e\}, n=25$, and let $V$ be the profile containing: 3 votes $a b c d e, 2$ votes $b c a d e, 2$ votes $c a b d e, 2$ votes $e d a b c, 2$ votes $e d b c a, 2$ votes $e d c a b, 2$ votes $a d e b c$, 2 votes $d e a b c, 2$ votes $e a d b c, 2$ votes $c b a d e, 2$ votes $c b d e a, 2$ votes $c b e a d$. The weighted majority graph associated with $V$ is:

$$
\begin{array}{c|c|c|c|c|c}
n_{V} & a & b & c & d & e \\
\hline a & - & 15 & 11 & 15 & 11 \\
\hline b & 10 & - & 15 & 13 & 13 \\
\hline c & 14 & 10 & - & 13 & 13 \\
\hline d & 10 & 12 & 12 & - & 14 \\
\hline e & 14 & 12 & 12 & 11 & -
\end{array}
$$

The Borda scores are respectively 52 for $a$ (Borda winner), 51 for $b, 50$ for $c, 48$ for $d$ and 49 for $e$.

For all $x \in X$, let

$$
S_{M W A, W}(x)=S_{B}(P, x)+\sum_{y \neq z \neq x} \max \left(N_{P}(y, z), N_{P}(z, y)\right) .
$$

We get

$$
\begin{aligned}
& S_{M W A, W}(a)=52+81=133 ; \\
& S_{M W A, W}(b)=51+82=133 ; \\
& S_{M W A, W}(c)=50+84=134 ; \\
& S_{M W A, W}(d)=48+83=131 ; \\
& S_{M W A, W}(e)=49+85=134 .
\end{aligned}
$$

The co-winners are $c$ and $e$. 